\title{
Sharp bounds for the ratio of modified Bessel functions
}

\author{
Zhen-Hang Yang and Shen-Zhou Zheng
}

\begin{abstract}
Let $I_{\nu}(x)$ be the modified Bessel functions of the first kind of order $\nu$, and $S_{p, \nu}(x)=W_{\nu}(x)^{2}-2 p W_{\nu}(x)-x^{2}$ with $W_{\nu}(x)=$ $x I_{\nu}(x) / I_{\nu+1}(x)$. We achieve necessary and sufficient conditions for the inequality $S_{p, \nu}(x)<u$ or $S_{p, \nu}(x)>l$ to hold for $x>0$ by establishing the monotonicity of $S_{p, \nu}(x)$ in $x \in(0, \infty)$ with $\nu>-3 / 2$. In addition, the best parameters $p$ and $q$ are obtained to the inequality $W_{\nu}(x)<(>) p+\sqrt{x^{2}+q^{2}}$ for $x>0$. Our main achievements improve some known results, and it seems to answer an open problem recently posed by Hornik and Grün in [13].
\end{abstract}

Mathematics Subject Classification (2010). Primary 33C10; Secondary $39 \mathrm{~B} 62$.

Keywords. modified Bessel functions of the first kind of order $\nu$, the ratio of modified Bessel functions, monotonicity, sharp bounds.

\section{Introduction}

Bessel functions as the solutions of Bessel's equations occur frequently in advanced studies in applied mathematics, physics, and engineering. The modified Bessel function of the first kind of order $\nu$, denoted by $I_{\nu}(x)$ as usual (cf. [33, page 77]), is a particular solution of the following second-order differential equation:

$$
x^{2} y^{\prime \prime}(x)+x y^{\prime}(x)-\left(x^{2}+\nu^{2}\right) y(x)=0,
$$

which is explicitly expressed by the infinite series

$$
I_{\nu}(x)=\sum_{n=0}^{\infty} \frac{(x / 2)^{2 n+\nu}}{n ! \Gamma(\nu+n+1)}=\frac{(x / 2)^{\nu}}{\Gamma(\nu+1)} \sum_{n=0}^{\infty} \frac{(x / 2)^{2 n}}{n !(\nu+1)_{n}}
$$

This work was completed with the support of NSFC grant 11371050 and NSFC-ERC grant 11611530539. 
for any $x \in \mathbb{R}$ and $\nu \in \mathbb{R} \backslash\{-1,-2, \cdots\}$, where $(a)_{n}$ is the Pochhammer symbol defined by

$$
(a)_{n}=a(a+1) \cdots(a+n-1)=\frac{\Gamma(a+n)}{\Gamma(a)}
$$

for any $n \in \mathbb{N}$ with $(a)_{0}=1$ for $a \neq 0,-1,-2, \cdots$.

It follows from [33, page 79] that $I_{\nu}$ satisfies the recurrence relations

$$
\begin{aligned}
& x I_{\nu}^{\prime}(x)+\nu I_{\nu}(x)=x I_{\nu-1}(x), \\
& x I_{\nu}^{\prime}(x)-\nu I_{\nu}(x)=x I_{\nu+1}(x),
\end{aligned}
$$

which implies that

$$
\frac{x I_{\nu}^{\prime}(x)}{I_{\nu}(x)}=\frac{x I_{\nu-1}(x)}{I_{\nu}(x)}-\nu=\frac{x I_{\nu+1}(x)}{I_{\nu}(x)}+\nu .
$$

It is worth pointing out that the ratio $x I_{\nu}(x) / I_{\nu+1}(x)$ plays an important role in finite elasticity $[29,30]$ and epidemiological models [21, 22], while another ratio $I_{\nu+1}(x) / I_{\nu}(x)$ has also appeared in probability and statistics $[10,27,12]$ with various applications in chemical kinetics $[2,18]$, optics [31] and signal processing [15]. For convenience, for any $x>0$ and $p+|q| \geq 0$ in the context we write by

$$
\begin{array}{ll}
W_{\nu}(x)=\frac{x I_{\nu}(x)}{I_{\nu+1}(x)}, & A_{p, q}(x)=p+\sqrt{x^{2}+q^{2}}, \\
R_{\nu}(x)=\frac{I_{\nu+1}(x)}{I_{\nu}(x)}, & G_{p, q}(x)=\frac{x}{p+\sqrt{x^{2}+q^{2}}} .
\end{array}
$$

Obviously, $W_{\nu}(x)=x / R_{\nu}(x)$.

Amos in 1974 first showed the bounds $G_{p, q}(x)$ for the ratio $R_{\nu}(x)$ (cf. formulas (11) and (16) in [3]) that for $x, \nu \geq 0$ there hold

$$
\begin{aligned}
G_{\nu+1, \nu+1}(x) & <R_{\nu}(x)<G_{\nu, \nu+2}(x), \\
G_{\nu+1 / 2, \nu+3 / 2}(x) & <R_{\nu}(x)<G_{\nu+1 / 2, \nu+1 / 2}(x) .
\end{aligned}
$$

For this reason, $G_{p, q}(x)$ is called Amos type bound for $R_{\nu}(x)$ by Hornik and Grün in [13]. For $\nu>-1$ and $p+|q| \geq 0$ it is easily seen that

$$
W_{\nu}(x)<(>) A_{p, q}(x) \Longleftrightarrow R_{\nu}(x)>(<) G_{p, q}(x) .
$$

So, one also calls $A_{p, q}(x)$ as Amos type bound for $W_{\nu}(x)$, and these inequalities (1.7) above are called Amos type ones.

In 1984 Simpson and Spector gave an alternative type inequality involving the ratio $W_{\nu}(x)$ as follows:

$$
W_{\nu}(x)^{2}-(2 \nu+1) W_{\nu}(x)-\left(x^{2}+\nu+\frac{1}{2}\right)>0, \quad \forall \nu \geq 0,
$$

for details to see Theorem 2 in [29]. For this, such an inequality similar to (1.8) is called as Simpson-Spector type inequality for $W_{\nu}(x)$. It is clear that 
Simpson-Spector type inequality (1.8) can be written that for $\nu \geq 0$,

$$
A_{\nu+1 / 2, \sqrt{(\nu+1 / 2)(\nu+3 / 2)}}(x)=\nu+\frac{1}{2}+\sqrt{x^{2}+\left(\nu+\frac{1}{2}\right)\left(\nu+\frac{3}{2}\right)}<W_{\nu}(x) .
$$

We would like to remark that Neuman in [24, Proposition 5] presented another Simpson-Spector type inequality for $W_{\nu}(x)$ as follows:

$$
W_{\nu}(x)^{2}-(2 \nu+1) W_{\nu}(x)-\left(x^{2}+\nu+\frac{1}{2}\right)<\nu+\frac{3}{2}, \quad \forall \nu>-\frac{3}{2},
$$

which extended the range of order $\nu$ from $[0, \infty)$ to $(-1, \infty)$ such that the first inequality of (1.6) holds. A companion one of (1.10) is due to Baricz and Neuman (cf. [4, Theorem 2.2]):

$$
W_{\nu}(x)^{2}-2 \nu W_{\nu}(x)-x^{2}>4(\nu+1), \quad \text { for all } \nu>-2,
$$

which indicates that the second inequality in (1.5) holds for $\nu>-1$.

Recently, Hornik and Grün [13] systematically investigated the lower and upper bounds for the modified Bessel functions ratio $R_{\nu}=I_{\nu+1} / I_{\nu}$ based on various results mentioned above and other involving achievements for examples [23], [36, E1. (A.5)], [17, Theorem 1.1], [28, Formulas (22) and (61)], [16]. They showed that the lower bound in (1.6) and upper bound in (1.5) for $\nu>-1$ are the best, and further extended the range of the inequality (1.9) from $\nu \geq 0$ to $\nu \geq-1 / 2$. Moreover, they pointed out that the range of $-1<\nu<-1 / 2$ deserves further investigation such that the inequality $R_{\nu}(x)<(>) G_{p, q}(x)$ holds for $x>0$.

Other results concerning Amos type inequality or Simpson-Spector type inequality can be found in [25], [5], [6], [7], [8] and references therein..

Motivated by Hornik and Grün's work and recent results mentioned above, the main aim of this paper is to study the monotonicity of the function

$$
x \mapsto S_{p, \nu}(x)=W_{\nu}(x)^{2}-2 p W_{\nu}(x)-x^{2}
$$

on $(0, \infty)$ for $\nu>-3 / 2$ by way of some power series expressions, and provide the necessary and sufficient conditions for the Simpson-Spector type inequality $S_{p, \nu}(x)<u$ or $S_{p, \nu}(x)>l$ for any $x>0$. The second aim is to determine the best parameters $p$ and $q$ such that the Amos type inequality $W_{\nu}(x)<(>) A_{p, q}(x)$ holds for $x \in(0, \infty)$, which in fact give new proofs of those inequalities mentioned previously and answers an open problem posted by Hornik and Grün [13].

The rest of the paper is organized as follows. We first give some auxiliary lemmas in Section 2. In Section 3 we are devoted to dealing with the monotonicity of $S_{p, \nu}(x)$ in accordance with the different ranges of $p$, and use it to establish the necessary and sufficient conditions such that SimpsonSpector type inequalities hold for $\nu>-3 / 2$. In the last section we give sharp constants $p$ and $q$ satisfying the Amos type inequality $W_{\nu}(x)<(>) A_{p, q}(x)$ for $\nu>-3 / 2$, and present some new Amos type bounds $G_{p, q}(x)$ for $R_{\nu}(x)$ in the case of $-1<\nu<-1 / 2$. 


\section{Some lemmas}

In order to prove our results, we need present some auxiliary lemmas. The first lemma is crucial which first appeared in [32, (3.5)] (see also [14]).

Lemma 2.1. Let $I_{\nu}$ be the modified Bessel functions of the first kind of order $\nu$ given by (1.2). Then we have

$$
\begin{gathered}
I_{u}(x) I_{\nu}(x)=\frac{1}{\Gamma(u+1) \Gamma(\nu+1)} \sum_{n=0}^{\infty} \frac{(u+\nu+n+1)_{n}}{n !(u+1)_{n}(\nu+1)_{n}}\left(\frac{x}{2}\right)^{2 n+u+\nu} \\
I_{\nu}(x)^{2}=\frac{1}{\Gamma(\nu+1)^{2}} \sum_{n=0}^{\infty} \frac{(2 \nu+n+1)_{n}}{n !(\nu+1)_{n}^{2}}\left(\frac{x}{2}\right)^{2 n+2 \nu}
\end{gathered}
$$

The following two lemmas are powerful tools to treat the monotonicity of ratios between two power series.

Lemma 2.2. ([11]) Let $A(t)=\sum_{k=0}^{\infty} a_{k} t^{k}$ and $B(t)=\sum_{k=0}^{\infty} b_{k} t^{k}$ be two real power series converging on $(-r, r)$ for some $r>0$ with $b_{k}>0$ for all $k$. If the sequence $\left\{a_{k} / b_{k}\right\}$ is increasing (or decreasing) for all $k$, then the function $t \mapsto A(t) / B(t)$ is also increasing (or decreasing) on $(0, r)$.

Lemma 2.3. ([35, Corollary 2.3.], [34]) Let $A(t)=\sum_{k=0}^{\infty} a_{k} t^{k}$ and $B(t)=$ $\sum_{k=0}^{\infty} b_{k} t^{k}$ be two real power series converging on $\mathbb{R}$ with $b_{k}>0$ for all $k$. If for certain $m \in \mathbb{N}$, the non-constant sequence $\left\{a_{k} / b_{k}\right\}$ is increasing (or decreasing) for $0 \leq k \leq m$ and decreasing (or increasing) for $k>m$, then there is a unique $t_{0} \in(0, \infty)$ such that the function $A / B$ is increasing (or decreasing) on $\left(0, t_{0}\right)$ and decreasing (or increasing) on $\left(t_{0}, \infty\right)$.

Remark 2.4. The condition in [35, Corollary 2.3.] that "the non-constant sequence $\left\{a_{k} / b_{k}\right\}$ is increasing (or decreasing) for $0 \leq k \leq m$ and decreasing (or increasing) for $k \geq m$ " contains the two special cases: $a_{k} / b_{k}=a_{0} / b_{0}$ for $0 \leq k \leq m$ and $a_{k} / b_{k}=a_{m} / b_{m}$ for $k \geq m$. In the two cases, the conclusion of [35, Corollary 2.3.] is obviously not true. Consequently, the range of $k$ that " $0 \leq k \leq m$ " should be modified as " $0 \leq k<m$ ", or replaced " $k \geq m$ " by " $k>m$ ". The same modification should also apply to [35, Theorem 2.1 ].

Lemma 2.5. ([26, Problems 85, 94]) If two given sequences $\left\{a_{n}\right\}_{n \geq 0}$ and $\left\{b_{n}\right\}_{n \geq 0}$ satisfy the following conditions:

$$
b_{n}>0, \quad \sum_{n=0}^{\infty} b_{n} t^{n} \text { converges for all values of } t \text {, and } \lim _{n \rightarrow \infty} \frac{a_{n}}{b_{n}}=s ;
$$

then, $\sum_{n=0}^{\infty} a_{n} t^{n}$ must be convergent for all values of $t$ too, and

$$
\lim _{t \rightarrow \infty} \frac{\sum_{n=0}^{\infty} a_{n} t^{n}}{\sum_{n=0}^{\infty} b_{n} t^{n}}=s .
$$




\section{Monotonicity of $S_{p, \nu}$ and Simpson-Spector type inequalities}

In this section, we are devoted to investigating the monotonicity of $S_{p, \nu}(x)$ in accordance with the different ranges of $p$, and use it to attain SimpsonSpector type inequalities. Let

$$
\begin{aligned}
& f_{1}(x):=x^{2} I_{\nu}(x)^{2}-2 p x I_{\nu}(x) I_{\nu+1}(x)-x^{2} I_{\nu+1}(x)^{2}, \\
& f_{2}(x):=I_{\nu+1}(x)^{2} .
\end{aligned}
$$

Then $S_{p, \nu}(x)$ can be expressed by

$$
S_{p, \nu}(x)=\frac{x^{2} I_{\nu}(x)^{2}-2 p x I_{\nu}(x) I_{\nu+1}(x)-x^{2} I_{\nu+1}(x)^{2}}{I_{\nu+1}(x)^{2}}=\frac{f_{1}(x)}{f_{2}(x)} .
$$

Combining the formulas (2.1) and (2.2) yields

$$
\begin{aligned}
f_{1}(x)= & x^{2} I_{\nu}(x)^{2}-2 p x I_{\nu}(x) I_{\nu+1}(x)-x^{2} I_{\nu+1}(x)^{2} \\
= & \frac{4}{\Gamma(\nu+1)^{2}} \sum_{n=0}^{\infty} \frac{(2 \nu+n+1)_{n}}{n !(\nu+1)_{n}^{2}}\left(\frac{x}{2}\right)^{2 n+2 \nu+2} \\
& -\frac{4 p}{\Gamma(\nu+2) \Gamma(\nu+1)} \sum_{n=0}^{\infty} \frac{(2 \nu+n+2)_{n}}{n !(\nu+2)_{n}(\nu+1)_{n}}\left(\frac{x}{2}\right)^{2 n+2 \nu+2} \\
& -\left(\frac{x}{2}\right)^{2} \frac{4}{\Gamma(\nu+2)^{2}} \sum_{n=0}^{\infty} \frac{(2 \nu+n+3)_{n}}{n !\left(\frac{x}{2}\right)^{2 n+2 \nu+2}} \\
= & \frac{4}{\Gamma(\nu+1)^{2}} \frac{\nu-p+1}{\nu+1}\left(\frac{x^{2}}{4}\right)^{\nu+1}+\frac{4}{\Gamma(\nu+1)^{2}}\left(\frac{x^{2}}{4}\right)^{\nu+1} \\
& \times \sum_{n=1}^{\infty} \frac{(2 \nu+n+2)_{n}}{n !(\nu+1)_{n}^{2}} \frac{(2 \nu-2 p+1) n-(2 \nu+1)(p-\nu-1)}{(2 n+2 \nu+1)(n+\nu+1)}\left(\frac{x^{2}}{4}\right)^{n} \\
:= & \frac{1}{\Gamma(\nu+1)^{2}}\left(\frac{x^{2}}{4}\right)^{\nu+1} \sum_{n=0}^{\infty} a_{n}\left(\frac{x^{2}}{4}\right)^{n},
\end{aligned}
$$

where

$$
a_{n}=4 \frac{(2 \nu-2 p+1) n+(2 \nu+1)(\nu+1-p)}{(2 n+2 \nu+1)(n+\nu+1)} \frac{(2 \nu+n+2)_{n}}{n !(\nu+1)_{n}^{2}} .
$$

In a similar way, we have

$$
\begin{aligned}
f_{2}(x) & =I_{\nu+1}(x)^{2}=\frac{1}{\Gamma(\nu+1)^{2}} \sum_{n=0}^{\infty} \frac{(2 \nu+n+3)_{n}}{n !(\nu+1)_{n+1}^{2}}\left(\frac{x}{2}\right)^{2 n+2 \nu+2} \\
& =\frac{1}{\Gamma(\nu+1)^{2}}\left(\frac{x^{2}}{4}\right)^{\nu+1} \sum_{n=0}^{\infty} b_{n}\left(\frac{x^{2}}{4}\right)^{n}
\end{aligned}
$$

where

$$
b_{n}=\frac{2}{(n+\nu+1)(n+2 \nu+2)} \frac{(2 \nu+n+2)_{n}}{n !(\nu+1)_{n}^{2}} .
$$


Therefore

$$
S_{p, \nu}(x)=\frac{f_{1}(x)}{f_{2}(x)}=\frac{\frac{1}{\Gamma(\nu+1)^{2}}\left(\frac{x^{2}}{4}\right)^{\nu+1} \sum_{n=0}^{\infty} a_{n}\left(\frac{x^{2}}{4}\right)^{n}}{\frac{1}{\Gamma(\nu+1)^{2}}\left(\frac{x^{2}}{4}\right)^{\nu+1} \sum_{n=0}^{\infty} b_{n}\left(\frac{x^{2}}{4}\right)^{n}}=\frac{\sum_{n=0}^{\infty} a_{n}\left(x^{2} / 4\right)^{n}}{\sum_{n=0}^{\infty} b_{n}\left(x^{2} / 4\right)^{n}},
$$

and

$$
\frac{a_{n}}{b_{n}}=2 \frac{n+2 \nu+2}{2 n+2 \nu+1}((2 \nu-2 p+1) n+(2 \nu+1)(\nu+1-p)) .
$$

It is easily seen that

$$
S_{p, \nu}(0)=\lim _{x \rightarrow 0} \frac{f_{1}(x)}{f_{2}(x)}=\frac{a_{0}}{b_{0}}=4(\nu+1)(\nu+1-p),
$$

and from Lemma 2.5 it is deduced that

$$
S_{p, \nu}(\infty)=\lim _{x \rightarrow \infty} \frac{f_{1}(x)}{f_{2}(x)}=\lim _{n \rightarrow \infty} \frac{a_{n}}{b_{n}}=\left\{\begin{array}{cc}
-\infty, & \text { if } p>\nu+\frac{1}{2}, \\
\nu+\frac{1}{2}, & \text { if } p=\nu+\frac{1}{2} \\
\infty, & \text { if } p<\nu+\frac{1}{2} .
\end{array}\right.
$$

To determine the monotonicity of $S_{p, \nu}$, by Lemmas 2.2 and 2.3 it suffices to observe the monotonicity of the sequence $\left\{a_{n} / b_{n}\right\}$. To that end, we observe

$$
\frac{a_{n+1}}{b_{n+1}}-\frac{a_{n}}{b_{n}}=-2\left(p-h_{n}(\nu)\right),
$$

where

$$
h_{n}(\nu)=(2 \nu+1) \frac{2 n^{2}+4(\nu+1) n+\nu(2 \nu+3)}{(2 n+2 \nu+1)(2 n+2 \nu+3)} .
$$

A simple computation yields

$$
\begin{aligned}
h_{n+1}(\nu)-h_{n}(\nu) & =\frac{2(2 \nu+1)(2 \nu+3)}{(2 n+2 \nu+1)(2 n+2 \nu+3)(2 n+2 \nu+5)} \\
& = \begin{cases}>0, & \text { if } \nu>-1 / 2 \\
>0, & \text { if }-3 / 2<\nu<-1 / 2 \text { and } n=0, \\
<0, & \text { if }-3 / 2<\nu<-1 / 2 \text { and } n \geq 1,\end{cases}
\end{aligned}
$$

which shows that for $\nu>-1 / 2$,

$$
\nu=h_{0}(\nu)<h_{n}(\nu)<h_{\infty}(\nu)=\nu+\frac{1}{2}, \quad n \geq 0 ;
$$

and for $-3 / 2<\nu<-1 / 2$,

$$
\begin{aligned}
\nu & =h_{0}(\nu)<h_{n}(\nu)<h_{1}(\nu)=\frac{(2 \nu+1)(\nu+2)}{2 \nu+5}, n=0,1 \\
\nu+\frac{1}{2} & =h_{\infty}(\nu)<h_{n}(\nu)<h_{1}(\nu)=\frac{(2 \nu+1)(\nu+2)}{2 \nu+5}, \quad n \geq 1 .
\end{aligned}
$$

We are now in a position to discuss the monotonicity of $S_{p, \nu}$ in accordance with the different cases of $\nu$ and $p$.

Case 1. While $\nu \geq-1 / 2$, it can be divided into three subcases to discuss.

Subcase 1.1. If $p \geq \nu+1 / 2$, from relations (3.6) and (3.8) then it is clearly seen that $a_{n+1} / b_{n+1}-a_{n} / b_{n} \leq 0$ for all $n \geq 0$, which means that the sequence 
$\left\{a_{n} / b_{n}\right\}_{n \geq 0}$ is decreasing. By Lemma 2.2 it follows that $x \mapsto f_{1}(x) / f_{2}(x)$ is decreasing on $(0, \infty)$. Therefore

$$
\begin{aligned}
& \left.\begin{array}{c}
-\infty, \quad \text { if } p>\nu+\frac{1}{2} \\
\nu+\frac{1}{2}, \quad \text { if } p=\nu+\frac{1}{2}
\end{array}\right\}=\lim _{n \rightarrow \infty} \frac{a_{n}}{b_{n}}=\lim _{x \rightarrow \infty} \frac{f_{1}(x)}{f_{2}(x)}<\frac{f_{1}(x)}{f_{2}(x)} \\
& <\lim _{x \rightarrow 0} \frac{f_{1}(x)}{f_{2}(x)}=\frac{a_{0}}{b_{0}}=4(\nu+1)(\nu+1-p) .
\end{aligned}
$$

Subcase 1.2. If $p \leq \nu$, similarly we have $a_{n+1} / b_{n+1}-a_{n} / b_{n} \geq 0$ for $n \geq 0$, that is to say, then the sequence $\left\{a_{n} / b_{n}\right\}_{n \geq 0}$ is increasing. By Lemma 2.2 it follows that $x \mapsto f_{1}(x) / f_{2}(x)$ is increasing on $(0, \infty)$. Hence,

$$
4(\nu+1)(\nu-p+1)=\lim _{x \rightarrow 0} \frac{f_{1}(x)}{f_{2}(x)}<\frac{f_{1}(x)}{f_{2}(x)}<\lim _{x \rightarrow \infty} \frac{f_{1}(x)}{f_{2}(x)}=\infty .
$$

Subcase 1.3. If $\nu<p<\nu+1 / 2$, as mentioned previously then the sequence $\left\{h_{n}(\nu)\right\}_{n \geq 0}$ is increasing, so $\left\{p-h_{n}(\nu)\right\}_{n \geq 0}$ is decreasing. This together with

$$
p-h_{0}(\nu)=p-\nu>0 \text { and } p-h_{\infty}(\nu)=p-\left(\nu+\frac{1}{2}\right)<0
$$

reveals that there is an $n_{0} \geq 1$ such that $p-h_{n}(\nu)>0$ for $0 \leq n \leq n_{0}$, and $p-h_{n}(\nu)<0$ for $n \geq n_{0}$. Combining with (3.6) yields that the sequence $\left\{a_{n} / b_{n}\right\}$ is decreasing for $0 \leq n \leq n_{0}$ and increasing for $n \geq n_{0}$. By Lemma 2.3 , it is deduced that there is an $x_{0}>0$ such that $f_{1} / f_{2}$ is decreasing on $\left(0, x_{0}\right)$ and increasing on $\left(x_{0}, \infty\right)$. Thus

$$
\begin{gathered}
\lambda_{p, \nu}=\frac{f_{1}\left(x_{0}\right)}{f_{2}\left(x_{0}\right)}<\frac{f_{1}(x)}{f_{2}(x)}<\lim _{x \rightarrow 0} \frac{f_{1}(x)}{f_{2}(x)}=4(\nu+1)(\nu-p+1), \quad \forall x \in\left(0, x_{0}\right), \\
\lambda_{p, \nu}=\frac{f_{1}\left(x_{0}\right)}{f_{2}\left(x_{0}\right)} \leq \frac{f_{1}(x)}{f_{2}(x)}<\lim _{x \rightarrow \infty} \frac{f_{1}(x)}{f_{2}(x)}=\infty, \quad \forall x \in\left(x_{0}, \infty\right),
\end{gathered}
$$

which implies that

$$
\frac{f_{1}(x)}{f_{2}(x)} \geq \lambda_{p, \nu}, \quad \forall x \in(0, \infty) .
$$

We now summarize these results above. More precisely, we have

Theorem 3.1. Let $S_{p, \nu}$ be defined on $(0, \infty)$ by (1.12) for $\nu>-1 / 2$. Then we have

(i) If $p>\nu+1 / 2$, then the function $S_{p, \nu}$ is decreasing from $(0, \infty)$ onto $(-\infty, 4(\nu+1)(\nu+1-p))$.

(ii) If $p=\nu+1 / 2$, then the function $S_{p, \nu}$ is decreasing from $(0, \infty)$ onto $(\nu+1 / 2,2(\nu+1))$.

(iii) If $\nu<p<\nu+1 / 2$, then there is an $x_{0}>0$ such that $S_{p, \nu}$ is decreasing on $\left(0, x_{0}\right)$ and increasing on $\left(x_{0}, \infty\right)$, with the estimate

$$
\lambda_{p, \nu} \leq S_{p, \nu}(x)<\infty,
$$

where $\lambda_{p, \nu}=S_{p, \nu}\left(x_{0}\right), x_{0}$ is a unique solution of the equation $S_{p, \nu}(x)=0$ on $(0, \infty)$. 
(iv) If $p \leq \nu$, then one has that the function $S_{p, \nu}$ is increasing from $(0, \infty)$ onto $(4(\nu+1)(\nu+1-p), \infty)$.

Remark 3.2. It is well known that $W_{-1 / 2}(x)=x \operatorname{coth} x$, so we easily check that Theorem 3.1 is also true for $\nu=-1 / 2$.

Thanks to Theorem 3.1 together with the remark above, we immediately conclude the following statement.

Theorem 3.3. Let $\nu \geq-1 / 2$. Then we have

(i) $S_{p, \nu}(x)<u$ holds for all $x>0$ if and only if $u \geq 4(\nu+1)(\nu+1-p)$ and $p \geq \nu+1 / 2$;

(ii) $l<S_{p, \nu}(x)$ holds for all $x>0$ if and only if

$$
l \leq L_{1}(p, \nu)= \begin{cases}\nu+\frac{1}{2}, & \text { if } p=\nu+\frac{1}{2}, \\ \lambda_{p, \nu}>0, & \text { if } \nu<p<\nu+\frac{1}{2}, \\ 4(\nu+1)(\nu+1-p), & \text { if } p \leq \nu,\end{cases}
$$

where $\lambda_{p, \nu}=S_{p, \nu}\left(x_{0}\right)$, and $x_{0}$ is a unique solution of the equation $S_{p, \nu}(x)=$ 0 on $(0, \infty)$.

Case 2. While $-3 / 2<\nu<-1 / 2$, as shown previously the sequence $\left\{h_{n}(\nu)\right\}_{n \geq 0}$ is increasing for $n=0,1$ and decreasing for $n \geq 1$. Then we have

$$
h_{0}(\nu)=\nu<\nu+\frac{1}{2}=h_{\infty}(\nu)<h_{n}(\nu) \leq h_{1}(\nu)=\frac{(2 \nu+1)(\nu+2)}{2 \nu+5} .
$$

We now distinguish four subcases to discuss.

Subcase 2.1. If $p \geq \max _{n \geq 0}\left(h_{n}(\nu)\right)=(2 \nu+1)(\nu+2) /(2 \nu+5)$, from relations (3.6), (3.9) and (3.10) we clearly see that $a_{n+1} / b_{n+1}-a_{n} / b_{n} \leq 0$ for $n \geq 0$, that is, the sequence $\left\{a_{n} / b_{n}\right\}_{n \geq 0}$ is decreasing, and so is $f_{1} / f_{2}$ on $(0, \infty)$ due to Lemma 2.2. Therefore

$$
-\infty=\lim _{x \rightarrow \infty} \frac{f_{1}(x)}{f_{2}(x)}<\frac{f_{1}(x)}{f_{2}(x)}<\lim _{x \rightarrow 0} \frac{f_{1}(x)}{f_{2}(x)}=\frac{a_{0}}{b_{0}}=4(\nu+1)(\nu+1-p)
$$

for all $x>0$.

Subcase 2.2. If $p \leq \min _{n \geq 0}\left(h_{n}(\nu)\right)=\nu$, then we clearly have $a_{n+1} / b_{n+1}-$ $a_{n} / b_{n} \geq 0$ for $n \geq 0$, which implies that the sequence $\left\{a_{n} / b_{n}\right\}_{n \geq 0}$ is increasing, and so is $f_{1} / f_{2}$ on $(0, \infty)$ due to Lemma 2.2. It follows that

$$
4(\nu+1)(\nu+1-p)=\frac{a_{0}}{b_{0}}=\lim _{x \rightarrow 0} \frac{f_{1}(x)}{f_{2}(x)}<\frac{f_{1}(x)}{f_{2}(x)}<\lim _{x \rightarrow \infty} \frac{f_{1}(x)}{f_{2}(x)}=\infty
$$

hold for all $x>0$.

Subcase 2.3. If $\nu=h_{0}(\nu)<p \leq h_{\infty}(\nu)=\nu+1 / 2$, from (3.6), (3.9) and (3.10) then we have

$$
\begin{aligned}
& \frac{a_{1}}{b_{1}}-\frac{a_{0}}{b_{0}}=-2(p-\nu)<0 \\
& \frac{a_{n+1}}{b_{n+1}}-\frac{a_{n}}{b_{n}}=-2\left[p-h_{n}(\nu)\right]>0, \quad \text { for } n \geq 1 .
\end{aligned}
$$


This shows that the sequence $\left\{a_{n} / b_{n}\right\}_{n \geq 0}$ is decreasing only for $n=0,1$; and increasing for $n \geq 1$. By Lemma 2.3 there exists an $x_{0}>0$ such that $f_{1} / f_{2}$ is decreasing on $\left(0, x_{0}\right)$ and increasing on $\left(x_{0}, \infty\right)$, and so we have that for $x \in\left(0, x_{0}\right)$,

$$
\lambda_{p, \nu}=\frac{f_{1}\left(x_{0}\right)}{f_{2}\left(x_{0}\right)}<\frac{f_{1}(x)}{f_{2}(x)}<\lim _{x \rightarrow 0} \frac{f_{1}(x)}{f_{2}(x)}=4(\nu+1)(\nu+1-p)
$$

and for $x \in\left(x_{0}, \infty\right)$,

$$
\lambda_{p, \nu}=\frac{f_{1}\left(x_{0}\right)}{f_{2}\left(x_{0}\right)}<\frac{f_{1}(x)}{f_{2}(x)}<\lim _{x \rightarrow \infty} \frac{f_{1}(x)}{f_{2}(x)}= \begin{cases}\nu+\frac{1}{2}, & \text { if } p=\nu+1 / 2 \\ \infty, & \text { if } \nu<p<\nu+1 / 2\end{cases}
$$

or

$$
\lambda_{p, \nu} \leq \frac{f_{1}(x)}{f_{2}(x)}<\left\{\begin{array}{cl}
2 \nu+2, & \text { if } p=\nu+1 / 2 \\
\infty, & \text { if } \nu<p<\nu+1 / 2 .
\end{array}\right.
$$

Subcase 2.4. If $\nu+1 / 2=h_{\infty}(\nu)<p<h_{1}(\nu)=(2 \nu+1)(\nu+2) /(2 \nu+5)$, from (3.13) we see that the sequence $\left\{a_{n} / b_{n}\right\}$ is decreasing for $n=0,1$. Note that $\left\{h_{n}(\nu)\right\}_{n \geq 1}$ is decreasing, so $\left\{p-h_{n}(\nu)\right\}_{n \geq 1}$ is increasing, which together with the facts that

$$
p-h_{1}(\nu)=p-\frac{(2 \nu+1)(\nu+2)}{2 \nu+5}<0 \text { and } p-h_{\infty}(\nu)=p-\left(\nu+\frac{1}{2}\right)>0
$$

reveals that there is an $n_{1}>1$ such that $p-h_{n}(\nu)<0$ for $1 \leq n \leq n_{1}$, and $p-h_{n}(\nu)>0$ for $n \geq n_{1}$. Combining (3.6) we see that the sequence $\left\{a_{n} / b_{n}\right\}$ is increasing for $1 \leq n \leq n_{1}$ and decreasing for $n \geq n_{1}$. It thus can be seen that the sequence $\left\{a_{n} / b_{n}\right\}$ is decreasing for $n=0,1$ and increasing for $1 \leq n \leq n_{0}$ then decreasing for $n \geq n_{0}$.

Obviously, we are not able to describe the monotone pattern of $f_{1} / f_{2}$ by directly using Lemmas 2.2 and 2.3. However, we can show that

$$
-\infty<\frac{f_{1}(x)}{f_{2}(x)}<\lim _{x \rightarrow 0} \frac{f_{1}(x)}{f_{2}(x)}=\frac{a_{0}}{b_{0}}, \quad \forall x>0 .
$$

In fact, for any $n \geq 1$ we have

$$
\begin{aligned}
& \frac{a_{n}}{b_{n}}-\frac{a_{0}}{b_{0}} \\
= & \frac{2(n+2 \nu+2)}{2 n+2 \nu+1}((2 \nu-2 p+1) n+(2 \nu+1)(\nu+1-p))-4(\nu+1)(\nu+1-p) \\
= & -\frac{2 n}{2 n+2 \nu+1}(p(2 n+2 \nu+1)-(2 \nu+1) n-(\nu+1)(2 \nu-1)) \\
< & -\frac{2 n}{2 n+2 \nu+1}\left[\left(\left(\nu+\frac{1}{2}\right)(2 n+2 \nu+1)-(2 \nu+1) n-(\nu+1)(2 \nu-1)\right)\right] \\
= & -n \frac{2 \nu+3}{2 n+2 \nu+1}<0,
\end{aligned}
$$

where the inequality holds due to $-3 / 2<\nu<-1 / 2$ and $\nu+1 / 2<p<$ $(2 \nu+1)(\nu+2) /(2 \nu+5)$. This implies that $a_{n} / b_{n} \leq a_{0} / b_{0}$ for any $n \geq 0$. 
Since $b_{n}>0$ for $n \geq 0$, we have

$$
\frac{f_{1}(x)}{f_{2}(x)}=\frac{\sum_{n=0}^{\infty} a_{n}\left(x^{2} / 4\right)^{n}}{\sum_{n=0}^{\infty} b_{n}\left(x^{2} / 4\right)^{n}}<\frac{\sum_{n=0}^{\infty}\left(a_{0} / b_{0}\right) b_{n}\left(x^{2} / 4\right)^{n}}{\sum_{n=0}^{\infty} b_{n}\left(x^{2} / 4\right)^{n}}=\frac{a_{0}}{b_{0}} .
$$

On the other hand, it is evident that

$$
\lim _{x \rightarrow \infty} \frac{f_{1}(x)}{f_{2}(x)}=\lim _{n \rightarrow \infty} \frac{a_{n}}{b_{n}}=\operatorname{sgn}(2 \nu-2 p+1) \infty=-\infty,
$$

which proves (3.14).

By summarizing the subcases 2.1-2.4, we conclude the following results.

Theorem 3.4. For $-3 / 2<\nu<-1 / 2$, let $S_{p, \nu}$ be defined by (1.12).

(i) If $p \geq(2 \nu+1)(\nu+2) /(2 \nu+5)$, then the function $S_{p, \nu}$ is decreasing from $(0, \infty)$ onto $(-\infty, 4(\nu+1)(\nu+1-p))$.

(ii) If $\nu+1 / 2<p<(2 \nu+1)(\nu+2) /(2 \nu+5)$, then we always have

$$
-\infty<S_{p, \nu}(x)<4(\nu+1)(\nu-p+1), \quad \forall x>0 .
$$

(iii) If $p=\nu+1 / 2$, then there exists an $x_{0}>0$ such that $S_{p, \nu}$ is decreasing on $\left(0, x_{0}\right)$ and increasing on $\left(x_{0}, \infty\right)$ with the estimates

$$
\lambda_{p, \nu} \leq S_{p, \nu}(x)<2 \nu+2, \quad \forall x>0,
$$

where $\lambda_{p, \nu}=S_{p, \nu}\left(x_{0}\right)$, and $x_{0}$ is a unique solution of the equation $S_{p, \nu}^{\prime}(x)=$ 0 on $(0, \infty)$.

(iv) If $\nu<p<\nu+1 / 2$, then there is an $x_{0}>0$ such that $S_{p, \nu}$ is decreasing on $\left(0, x_{0}\right)$, and increasing on $\left(x_{0}, \infty\right)$ with

$$
\lambda_{p, \nu} \leq S_{p, \nu}(x)<\infty, \quad \forall x>0,
$$

where $\lambda_{p, \nu}=S_{p, \nu}\left(x_{0}\right)$, and $x_{0}$ is a unique solution of the equation $S_{p, \nu}^{\prime}(x)=$ 0 on $(0, \infty)$.

(v) If $p \leq \nu$, then one has that the function $S_{p, \nu}$ is increasing from $(0, \infty)$ onto $(4(\nu+1)(\nu+1-p), \infty)$.

Theorem 3.5. Let $-3 / 2<\nu<-1 / 2$. Then we have

(i) the inequality $S_{p, \nu}(x)<u$ holds for all $x>0$ if and only if $u \geq$ $4(\nu+1)(\nu+1-p)$ and $p \geq \nu+1 / 2$;

(ii) the inequality $l<S_{p, \nu}(x)$ holds for all $x>0$ if and only if

$$
l \leq L_{2}(p, \nu)= \begin{cases}\lambda_{p, \nu}, & \text { if } \nu<p \leq \nu+\frac{1}{2}, \\ 4(\nu+1)(\nu+1-p), & \text { if } p \leq \nu,\end{cases}
$$

where $\lambda_{p, \nu}=S_{p, \nu}\left(x_{0}\right)$, and $x_{0}$ is a unique solution of the equation $S_{p, \nu}^{\prime}(x)=$ 0 on $(0, \infty)$.

On the basis of Theorems 3.3 and 3.5, we immediately obtain the following corollary.

Corollary 3.6. Let $\nu>-3 / 2$. Then the inequality $S_{p, \nu}(x)<u$ holds for all $x>0$ if and only if $u \geq 4(\nu+1)(\nu+1-p)$ and $p \geq \nu+1 / 2$. 
Remark 3.7. In particular, by taking $p=\nu+1 / 2$ and $u=4(\nu+1)(\nu+1-p)$ we deduces (1.10) which was first proved in [24, Proposition 5].

Corollary 3.8. Let $\nu>-3 / 2$. Then the inequality $l<S_{p, \nu}(x)$ holds for all $x>0$ if and only if

$$
l \leq L(p, \nu)= \begin{cases}\nu+\frac{1}{2}, & \text { if } p=\nu+\frac{1}{2}, \nu>-\frac{1}{2}, \\ \lambda_{p, \nu}, & \text { if } p=\nu+\frac{1}{2}, \frac{3}{2}<\nu<-\frac{1}{2}, \\ \lambda_{p, \nu}, & \text { if } \nu<p<\nu+\frac{1}{2}, \\ 4(\nu+1)(\nu+1-p), & \text { if } p \leq \nu,\end{cases}
$$

where $\lambda_{p, \nu}=S_{p, \nu}\left(x_{0}\right)$, and $x_{0}$ is a unique solution of the equation $S_{p, \nu}^{\prime}(x)=$ 0 on $(0, \infty)$.

Remark 3.9. Taking $p=\nu+1 / 2$ and $l=L(p, \nu)$ for $\nu>-1 / 2$ in Corollary 3.8 , we derive inequality (1.8) proved in [29]. Letting $p=\nu$ and $l=L(p, \nu)$ yields inequality (1.11) for $\nu>-3 / 2$. We claim that inequality (1.11) is valid for $\nu>-2$, which suffices to show that the sequence $\left\{a_{n} / b_{n}\right\}_{n \geq 0}$ is increasing for $\nu>-2$ by Lemma 2.2. Indeed, if $p=\nu>-2$ then we have

$$
b_{0}=\frac{1}{(\nu+1)^{2}}>0, \quad b_{1}=\frac{2}{(\nu+1)^{2}(\nu+2)}>0
$$

and $b_{n}>0$ for $n \geq 2$, and

$$
\begin{aligned}
& \frac{a_{1}}{b_{1}}-\frac{a_{0}}{b_{0}}=0, \quad \frac{a_{2}}{b_{2}}-\frac{a_{1}}{b_{1}}=\frac{4}{2 \nu+5}>0, \\
& \frac{a_{n+1}}{b_{n+1}}-\frac{a_{n}}{b_{n}}=\frac{4 n(n+2 \nu+2)}{(2 n+2 \nu+1)(2 n+2 \nu+3)}>0 \text { for } n \geq 2 .
\end{aligned}
$$

\section{Amos type inequalities for $W_{\nu}(x)$}

In this section, we mainly are devoted to showing the necessary and sufficient conditions for the Amos type inequality

$$
W_{\nu}(x)=\frac{x I_{\nu}(x)}{I_{\nu+1}(x)}<(>) p+\sqrt{x^{2}+q^{2}}=A_{p, q}(x), \quad \forall x>0 .
$$

Similar to [13, Theorem 1], we have the following lemma.

Lemma 4.1. Let $\nu>-3 / 2$ and $p \in \mathbb{R}, q \geq 0$. If Amos type inequality (4.1) holds for all $x>0$, then it is necessary to ensure

$$
p \geq(\leq) \nu+\frac{1}{2}, \text { and } p+q \geq(\leq) 2(\nu+1) .
$$

Proof. Using the asymptotic formulas

$$
\begin{aligned}
& I_{\nu}(x) \sim\left(\frac{x}{2}\right)^{\nu} / \Gamma(\nu+1) \quad \text { as } x \rightarrow 0, \\
& I_{\nu}(x) \sim \frac{e^{x}}{\sqrt{2 \pi x}}\left(1-\frac{4 \nu^{2}-1}{1 !(8 x)}\right) \quad \text { as } x \rightarrow \infty
\end{aligned}
$$


listed in [1, page 375 and 377], we have

$$
\begin{aligned}
& \frac{x I_{\nu}(x)}{I_{\nu+1}(x)}-\left(p+\sqrt{x^{2}+q^{2}}\right) \sim \frac{x\left(\frac{x}{2}\right)^{\nu} / \Gamma(\nu+1)}{\left(\frac{x}{2}\right)^{\nu+1} / \Gamma(\nu+2)}-\left(p+\sqrt{x^{2}+q^{2}}\right) \\
& \longrightarrow 2(\nu+1)-(p+q), \quad \text { as } x \rightarrow 0,
\end{aligned}
$$

and

$$
\begin{aligned}
& \frac{x I_{\nu}(x)}{I_{\nu+1}(x)}-\left(p+\sqrt{x^{2}+q^{2}}\right) \sim \frac{x \frac{e^{x}}{\sqrt{2 \pi x}}\left(1-\frac{4 \nu^{2}-1}{8 x}\right)}{\frac{e^{x}}{\sqrt{2 \pi x}}\left(1-\frac{4(\nu+1)^{2}-1}{8 x}\right)}-\left(p+\sqrt{x^{2}+q^{2}}\right) \\
& =\frac{x\left(8 x-4 \nu^{2}+1\right)}{8 x-(2 \nu+3)(2 \nu+1)}-p-\sqrt{x^{2}+q^{2}} \longrightarrow \nu+\frac{1}{2}-p, \quad \text { as } x \rightarrow \infty .
\end{aligned}
$$

Therefore, it is an important observation that if the inequality (4.1) holds for all $x>0$, then we get

$$
-(p+q) \leq(\geq) 0 \text { and } \nu+\frac{1}{2}-p \leq(\geq) 0,
$$

which proves the desired assertion.

Lemma 4.2. For any $\nu>-2$, the function $x \mapsto W_{\nu}(x)$ is increasing from $(0, \infty)$ onto $(2 \nu+2, \infty)$.

Proof. The monotonicity of $W_{\nu}$ on $(0, \infty)$ has been proven in $[4$, Theorem $2.2]$, and it suffices to show $W_{\nu}\left(0^{+}\right)=2 \nu+2$ and $W_{\nu}(\infty)=\infty$, which easily follow from the asymptotic formulas (4.2) and (4.3). In fact, utilizing the expansion (1.2) we have

$$
\begin{aligned}
& W_{\nu}(x)=\frac{x I_{\nu}(x)}{I_{\nu+1}(x)} \sim \frac{x(x / 2)^{\nu} / \Gamma(\nu+1)}{(x / 2)^{\nu+1} / \Gamma(\nu+2)}=2(\nu+1) \text { as } x \rightarrow 0, \\
& W_{\nu}(x)=\frac{x I_{\nu}(x)}{I_{\nu+1}(x)} \sim x \rightarrow \infty \text { as } x \rightarrow \infty .
\end{aligned}
$$

4.1. The necessary and sufficient conditions for $W_{\nu}(x)<(>) A_{p, q}(x)$

Theorem 4.3. Let $\nu>-3 / 2$. Then the the following inequality

$$
W_{\nu}(x)<p+\sqrt{x^{2}+p^{2}+u}=A_{p, \sqrt{p^{2}+u}}(x)
$$

holds for all $x>0$ if and only if $(p, u) \in \Omega$ with

$$
\Omega=\left\{\nu+\frac{1}{2} \leq p \leq 2(\nu+1), u \geq 4(\nu+1)(\nu+1-p)\right\} \cup\left\{p>2(\nu+1), u \geq-p^{2}\right\} .
$$

Furthermore, for all $x>0$ we have

$$
\min _{(p, u) \in \Omega} A_{p, \sqrt{p^{2}+u}}(x)=\nu+\frac{1}{2}+\sqrt{x^{2}+\left(\nu+\frac{3}{2}\right)^{2}} .
$$


Proof. If the inequality (4.4) holds for all $x>0$, then by Lemma 4.1 we have

$$
(p, u) \in\left\{p \geq \nu+\frac{1}{2}, p^{2}+u \geq 0, p+\sqrt{p^{2}+u} \geq 2(\nu+1)\right\}:=D_{1} .
$$

Hence, it suffices to show $D_{1}=\Omega$. Indeed, $D_{1}$ can be written as

$$
\begin{aligned}
D_{1}= & \left\{\nu+\frac{1}{2} \leq p \leq 2(\nu+1), p^{2}+u \geq 0, p+\sqrt{p^{2}+u} \geq 2(\nu+1)\right\} \\
& \cup\left\{p \geq \max \left(\nu+\frac{1}{2}, 2(\nu+1)\right), p^{2}+u \geq 0, p+\sqrt{p^{2}+u} \geq 2(\nu+1)\right\} \\
:= & D_{11} \cup D_{12} .
\end{aligned}
$$

It is obvious that

$$
D_{12}=\left\{p>2(\nu+1), p^{2}+u \geq 0\right\} .
$$

While $p \leq 2(\nu+1)$, the inequality $p+\sqrt{p^{2}+u} \geq 2(\nu+1)$ is equivalent to

$$
u \geq 4(\nu+1)(\nu+1-p)
$$

which implies

$$
p^{2}+u \geq p^{2}+4(\nu+1)(\nu+1-p)=(2 \nu+2-p)^{2} \geq 0 .
$$

Therefore,

$$
D_{11}=\left\{\nu+\frac{1}{2} \leq p \leq 2(\nu+1), u \geq 4(\nu+1)(\nu+1-p)\right\},
$$

which realizes the necessity.

Let us now prove the sufficiency. If $(p, u) \in D_{11}$, that is, $\nu+1 / 2 \leq p \leq$ $2(\nu+1)$ and $u \geq 4(\nu+1)(\nu+1-p)$, by considering

$$
S_{p, \nu}(x)=\left(W_{\nu}(x)-p+\sqrt{x^{2}+p^{2}+u}\right)\left(W_{\nu}(x)-p-\sqrt{x^{2}+p^{2}+u}\right)
$$

and $W_{\nu}(x)>2(\nu+1) \geq p$ due to Lemma 4.2 , we have $W_{\nu}(x)-p+$ $\sqrt{x^{2}+p^{2}+u}>0$ for all $x>0$. This means that the inequality $S_{p, \nu}(x)<u$ holds for all $x>0$ is equivalent to $W_{\nu}(x)<A_{p, \sqrt{p^{2}+u}}(x)$ for all $x>0$ due to Theorem 3.6.

On the other hand, we claim that

$$
\min _{(p, u) \in D_{11}} A_{p, \sqrt{p^{2}+u}}(x)=A_{\nu+1 / 2, \nu+3 / 2}(x)=\nu+\frac{1}{2}+\sqrt{x^{2}+\left(\nu+\frac{3}{2}\right)^{2}} .
$$

In fact, for the case of $(p, u) \in D_{11}$ we get

$$
\begin{aligned}
A_{p, \sqrt{p^{2}+u}}(x) & =p+\sqrt{x^{2}+p^{2}+u} \geq p+\sqrt{x^{2}+p^{2}+4(\nu+1)^{2}-4(\nu+1) p} \\
& =p+\sqrt{x^{2}+(2 \nu+2-p)^{2}}:=B_{p}(x) .
\end{aligned}
$$

It is easy to check that $p \mapsto B_{p}(x)$ is increasing on $\mathbb{R}$, then we have

$$
B_{p}(x) \geq B_{\nu+1 / 2}(x)=\nu+\frac{1}{2}+\sqrt{x^{2}+\left(\nu+\frac{3}{2}\right)^{2}}=A_{\nu+1 / 2, \nu+3 / 2}(x) \text {. }
$$


To our aim, it remains to prove that (4.4) holds for all $x>0$ if $(p, u) \in$ $D_{12}=\left\{p>2(\nu+1), p^{2}+u \geq 0\right\}$. It is easy to see that

$$
A_{p, \sqrt{p^{2}+u}}(x)=p+\sqrt{x^{2}+p^{2}+u}>2(\nu+1)+x,
$$

which implies

$$
\min _{(p, u) \in D_{12}} A_{p, \sqrt{p^{2}+u}}(x)=2(\nu+1)+x .
$$

A simple computation gives

$$
\begin{aligned}
& \min _{(p, u) \in D_{12}} A_{p, \sqrt{p^{2}+u}}(x)-\min _{(p, u) \in D_{11}} A_{p, \sqrt{p^{2}+u}}(x) \\
= & 2(\nu+1)+x-\left(\nu+\frac{1}{2}+\sqrt{x^{2}+\left(\nu+\frac{3}{2}\right)^{2}}\right) \\
= & x+\left(\nu+\frac{3}{2}\right)-\sqrt{x^{2}+\left(\nu+\frac{3}{2}\right)^{2}}>0 .
\end{aligned}
$$

Then we conclude that for $(p, u) \in D_{12}$, the inequality $W_{\nu}(x)<A_{p, \sqrt{p^{2}+u}}(x)$ also holds for all $x>0$. This also proves (4.5) and the proof is completed. follows.

Setting $p^{2}+u=q^{2}$, the above theorem can be equivalently stated as

Theorem 4.4. Let $\nu>-3 / 2$ and $p \in \mathbb{R}, q \geq 0$. Then the inequality

$$
W_{\nu}(x)<p+\sqrt{x^{2}+q^{2}}=A_{p, q}(x)
$$

holds for all $x>0$ if and only if $(p, q) \in \Omega^{*}$, where

$$
\Omega^{*}=\left\{p \geq \nu+\frac{1}{2} \text { and } p+q \geq 2(\nu+1)\right\} \text {. }
$$

Furthermore, we have

$$
\min _{(p, q) \in \Omega^{*}} A_{p, q}(x)=A_{v+1 / 2, v+3 / 2}(x) .
$$

Remark 4.5. Clearly, when $\nu>-1$ and $p+q \geq 0$, Theorem 4.4 implies that another Amos type inequality $R_{\nu}(x)>G_{p, q}(x)$ holds for $x>0$ if and only if $(p, q) \in \Omega^{*}$ with $\max _{(p, q) \in \Omega^{*}} G_{p, q}(x)=G_{v+1 / 2, v+3 / 2}(x)$, which is Theorem 3 in [13]. Here, we in fact give a new proof of this theorem.

As shown in the proof of Theorem 4.3, if $p<2(\nu+1)$, then $W_{\nu}(x)-p+$ $\sqrt{x^{2}+p^{2}+u}>0$ for all $x>0$, which means that the inequality $l<S_{p, \nu}(x)$ is equivalent to $A_{p, \sqrt{p^{2}+l}}(x)<W_{\nu}(x)$ if $p^{2}+l \geq 0$. Therefore, from Theorem 3.8 we immediately get

Theorem 4.6. Let $\nu>-3 / 2$. Then the following inequality

$$
A_{p, \sqrt{p^{2}+l}}(x)=p+\sqrt{x^{2}+p^{2}+l}<W_{\nu}(x)
$$


holds for all $x>0$ if and only if $(p, l) \in \Delta_{1} \cup \Delta_{2} \cup \Delta_{3}$, where

$$
\begin{aligned}
& \Delta_{1}:=\left\{-\left(\nu+\frac{1}{2}\right)^{2} \leq l \leq \nu+\frac{1}{2}, p=\nu+\frac{1}{2}, \nu \geq-\frac{1}{2}\right\}, \\
& \Delta_{2}:=\left\{-p^{2} \leq l \leq \lambda_{p, \nu}, \nu<p<\nu+\frac{1}{2}\right\}, \\
& \Delta_{3}:=\left\{-p^{2} \leq l \leq 4(\nu+1)(\nu+1-p), p \leq \nu\right\}
\end{aligned}
$$

with $\lambda_{p, \nu}=S_{p, \nu}\left(x_{0}\right)$, and $x_{0}$ is a unique solution of the equation $S_{p, \nu}^{\prime}(x)=0$ on $(0, \infty)$ with $p^{2}+\lambda_{p, \nu} \geq 0$ for $\nu<p<\nu+1 / 2$. Moreover,

$$
\begin{gathered}
\max _{(p, l) \in \Delta_{1}} A_{p, \sqrt{p^{2}+l}}(x)=\nu+\frac{1}{2}+\sqrt{x^{2}+\left(\nu+\frac{1}{2}\right)\left(\nu+\frac{3}{2}\right)}, \\
\max _{(p, l) \in \Delta_{3}} A_{p, \sqrt{p^{2}+l}}(x)=\nu+\sqrt{x^{2}+(\nu+2)^{2}} .
\end{gathered}
$$

Proof. By Lemma 4.1, a necessary condition for the inequality $A_{p, \sqrt{p^{2}+l}}(x)<$ $W_{\nu}(x)$ to hold for all $x>0$ is stated to be

$$
\begin{aligned}
(p, l) & \in\left\{p \leq \nu+\frac{1}{2}, p^{2}+l \geq 0, p+\sqrt{x^{2}+p^{2}+l} \leq 2(\nu+1)\right\} \\
& =\left\{p \leq \nu+\frac{1}{2}, p^{2}+l \geq 0, l \leq 4(\nu+1)(\nu+1-p)\right\}:=D_{2} .
\end{aligned}
$$

Let

$$
\begin{aligned}
& \Delta_{11}:=\left\{l \leq \nu+\frac{1}{2}, p=\nu+\frac{1}{2}, \nu \geq-\frac{1}{2}\right\}, \\
& \Delta_{12}:=\left\{l \leq \lambda_{p, \nu}, p=\nu+\frac{1}{2}, \frac{3}{2}<\nu<-\frac{1}{2}\right\}, \\
& \Delta_{2}^{\prime}:=\left\{l \leq \lambda_{p, \nu}, \nu<p<\nu+\frac{1}{2}\right\}, \\
& \Delta_{3}^{\prime}:=\{l \leq 4(\nu+1)(\nu+1-p), p \leq \nu\} .
\end{aligned}
$$

Then, by Theorem 3.8 the inequality $A_{p, \sqrt{p^{2}+l}}(x)<W_{\nu}(x)$ holds for all $x>0$ if and only if

$$
(p, l) \in\left(\Delta_{11} \cup \Delta_{12} \cup \Delta_{2}^{\prime} \cup \Delta_{3}^{\prime}\right) \cap D_{2} .
$$

(i) From (3.14) we see that $\lambda_{\nu+1 / 2}<\nu+1 / 2$ and

$$
p^{2}+l \leq\left(\nu+\frac{1}{2}\right)^{2}+\left(\nu+\frac{1}{2}\right)=\left(\nu+\frac{1}{2}\right)\left(\nu+\frac{3}{2}\right)<0
$$

for any $-3 / 2<\nu<-1 / 2$, which means that $\Delta_{12} \cap D_{2}=\Phi$. While $\Delta_{11} \cap D_{2}=$ $\Delta_{1}$ is obvious, hence $\left(\Delta_{11} \cup \Delta_{12}\right) \cap D_{2}=\Delta_{1}$. In addition, for all $(p, l) \in \Delta_{1}$ we have

$$
A_{p, \sqrt{p^{2}+l}}(x)=\nu+\frac{1}{2}+\sqrt{x^{2}+\left(\nu+\frac{1}{2}\right)^{2}+l} \leq \nu+\frac{1}{2}+\sqrt{x^{2}+\left(\nu+\frac{1}{2}\right)^{2}+\left(\nu+\frac{1}{2}\right)},
$$

which proves (4.8).

(ii) From (3.11) and (3.14) it reveals that $\lambda_{p, \nu}<4(\nu+1)(\nu+1-p)$, which 
indicates that $\Delta_{2}^{\prime} \cap D_{2}=\Delta_{2}$.

(iii) It is obvious that $\Delta_{3}^{\prime} \cap D_{2}=\Delta_{3}$. For all $(p, l) \in \Delta_{3}$, we deduce that

$$
\begin{aligned}
A_{p, \sqrt{p^{2}+l}}(x) & =p+\sqrt{x^{2}+p^{2}+l} \\
& \leq p+\sqrt{x^{2}+p^{2}+4(\nu+1)(\nu+1-p)}=B_{p}(x) .
\end{aligned}
$$

As mentioned in the proof of Theorem 4.3, the function $p \mapsto B_{p}(x)$ is increasing on $\mathbb{R}$, and therefore, for $p \leq \nu$,

$$
B_{p}(x) \leq B_{\nu}(x)=\nu+\sqrt{x^{2}+(\nu+2)^{2}},
$$

which proves (4.9). Thus we complete the proof of this theorem. follows.

Let $p^{2}+l=q^{2}$. Then the above theorem can be equivalently stated as

Theorem 4.7. Let $\nu>-3 / 2$ and $p \in \mathbb{R}, q \geq 0$. Then the following inequality

$$
A_{p, q}(x)=p+\sqrt{x^{2}+q^{2}}<W_{\nu}(x)
$$

holds for all $x>0$ if and only if $(p, q) \in \Delta_{1}^{*} \cup \Delta_{2}^{*} \cup \Delta_{3}^{*}$, where

$$
\begin{aligned}
& \Delta_{1}^{*}:=\left\{p=\nu+\frac{1}{2}, q \leq \sqrt{\left(\nu+\frac{1}{2}\right)\left(\nu+\frac{3}{2}\right)}, \nu \geq-\frac{1}{2}\right\}, \\
& \Delta_{2}^{*}:=\left\{\nu<p<\nu+\frac{1}{2}, p^{2}+\lambda_{p, \nu} \geq 0, q \leq \sqrt{p^{2}+\lambda_{p, \nu}}\right\}, \\
& \Delta_{3}^{*}:=\{p \leq \nu, q \leq 2 \nu+2-p\}
\end{aligned}
$$

here $\lambda_{p, \nu}=S_{p, \nu}\left(x_{0}\right)$, and $x_{0}$ is a unique solution of the equation $S_{p, \nu}^{\prime}(x)=0$ on $(0, \infty)$. Furthermore, we have

$$
\begin{aligned}
\max _{(p, q) \in \Delta_{1}^{*}} A_{p, q}(x) & =A_{v+1 / 2, \sqrt{(\nu+1 / 2)(\nu+3 / 2)}}(x), \\
\max _{(p, q) \in \Delta_{3}^{*}} A_{p, q}(x) & =A_{v, v+2}(x) .
\end{aligned}
$$

Remark 4.8. If the conditions " $\nu>-1$ and $p+q \geq 0 "$ are added to Theorem 4.7, then we deduce that another Amos type inequality $R_{\nu}(x)<G_{p, q}(x)$ holds for $x>0$ if and only if $(p, q) \in \Delta_{1}^{*} \cup \Delta_{2}^{*} \cup \Delta_{3}^{*}$.

Clearly, the assertions that inequality $R_{\nu}(x)<G_{p, q}(x)$ holds for $x>0$ if $(p, q) \in \Delta_{i}^{*}(i=1,2,3)$ correspond to Theorems $9,10(v \geq-1 / 2)$ and 6 in [13], respectively. From this it is easy to see that Theorem 4.7 under the conditions " $\nu>-1$ and $p+q \geq 0$ " improves Hornik and Grün's results in [13] and solves the open problem posted by them. we have

Additionally, letting $u, l=4(\nu+1)(\nu+1-p)$ in Theorems 4.3 and 4.6

Corollary 4.9. Let $\nu>-3 / 2$. Then the double inequality

$$
p_{1}+\sqrt{x^{2}+\left(2 \nu+2-p_{1}\right)^{2}}<W_{\nu}(x)<p_{2}+\sqrt{x^{2}+\left(2 \nu+2-p_{2}\right)^{2}}
$$

hold for $x>0$ if and only if $p_{1} \leq \nu$ and $p_{2} \geq \nu+1 / 2$. 
Remark 4.10. The above corollary contains two rational bounds for $W_{\nu}(x)$. Indeed, if taking $p_{1}=\nu,-\infty$ and $p_{2}=\nu+1 / 2,2 \nu+2$, then by the monotonicity of $p \mapsto B_{p}(x)$ mentioned in the proof of Theorem 4.3, we have

$2 \nu+2<\nu+\sqrt{x^{2}+(\nu+2)^{2}}<W_{\nu}(x)<\nu+\frac{1}{2}+\sqrt{x^{2}+\left(\nu+\frac{3}{2}\right)^{2}}<2 \nu+2+x$ for all $x>0$.

4.2. Some computable lower bounds $A_{p, q}(x)$ for $W_{\nu}(x)$ if $-3 / 2<\nu<p<$ $\nu+1 / 2$

Although the necessary and sufficient conditions for $W_{\nu}(x)>A_{p, q}(x)$ or $R_{\nu}(x)<G_{p, q}(x)$ to hold for $x>0$ have been given in Theorem 4.7, the maximal $q=\sqrt{p^{2}+\lambda_{p, \nu}}$ for $\nu<p<\nu+1 / 2$ is related to a variable $\lambda_{p, \nu}$. As shown in Section 3, $\lambda_{p, \nu}=S_{p, \nu}\left(x_{0}\right)$ for $\nu<p<\nu+1 / 2$, where $x_{0}$ is a unique solution of the equation $S_{p, \nu}^{\prime}(x)=0$ on $(0, \infty)$ and $\lambda_{p, \nu}<4(\nu+1)(\nu-p+1)$. In general, $\lambda_{p, \nu}$ is not computable, and it is of practical value to find some lower bounds for $\lambda_{p, \nu}$ by elementary functions.

In [13, Theorem 7], Hornik and Grün presented a class of new upper bounds $G_{p, q_{\nu}^{*}(p)}(x)$ for $R_{\nu}(x)$ for $-1<v<p<\min (v+1 / 2,2 v+1):=p_{\nu}^{b}$, where

$$
q_{\nu}^{*}(p)=\sqrt{2(\nu+1 / 2-p)}+\sqrt{(p+1)(2 \nu+1-p)} .
$$

It is undoubted that

$$
\begin{aligned}
& \left\{G_{p, q_{\nu}^{*}(p)}(x):-1<v<p<p_{\nu}^{b}\right\} \\
\subseteq & \left\{G_{p, \sqrt{p^{2}+\lambda_{p, \nu}}}(x):-1<\nu<p<\nu+\frac{1}{2}, p^{2}+\lambda_{p, \nu} \geq 0\right\},
\end{aligned}
$$

but we are not able to check it. In this subsection, by the definition of $\lambda_{p, \nu}$ and $a_{n} / b_{n}$ given in (3.3) we give some easily computable lower bounds $A_{p, q}(x)$ for $W_{\nu}(x)$ if $-3 / 2<\nu<p<\nu+1 / 2$, and compare with $A_{p, q_{\nu}^{*}(p)}(x)$ in the case of $v>-1$.

Corollary 4.11. Let $\nu \geq-1 / 2$. Then, for $\nu<p<\nu+1 / 2$ the inequality

$$
A_{p, \xi_{p}}(x)=p+\sqrt{x^{2}+\xi_{p}^{2}}<W_{\nu}(x)
$$

holds for all $x>0$ with

$$
\xi_{p}=\sqrt{(2 \nu+3-p)^{2}-(3 \nu+11 / 2)} ;
$$

For $\nu<p \leq(\nu+2)(2 \nu+1) /(2 \nu+5)<\nu+1 / 2$, we have

$$
A_{p, \theta_{p}}(x)=p+\sqrt{x^{2}+\theta_{p}^{2}}<W_{\nu}(x)
$$

for all $x>0$, where

$$
\theta_{p}=\sqrt{(2 \nu+3-p)^{2}-(2 \nu+5)}
$$


Proof. We fist prove that if $-1 / 2 \leq \nu<p<\nu+1 / 2$, then

$$
\frac{a_{n}}{b_{n}} \geq c(p)=(2 \nu+3)(2 \nu+1-2 p)+\nu+\frac{1}{2}>0
$$

hold for all $n \geq 0$. For this, we write $a_{n} / b_{n}$ given in (3.3) as

$$
\frac{a_{n}}{b_{n}}=(n+2 \nu+2)(2 \nu+1-2 p)+\left(\nu+\frac{1}{2}\right) \frac{2 n+4 \nu+4}{2 n+2 \nu+1} .
$$

Then, by a simple calculation we obtain

$$
\begin{aligned}
\frac{a_{0}}{b_{0}}-c(p) & =4(\nu+1)(\nu+1-p)-\left((2 \nu+3)(2 \nu+1-2 p)+\nu+\frac{1}{2}\right) \\
& =\frac{1}{2}(4 p-2 \nu+1)>\frac{1}{2}(4 \nu-2 \nu+1)=\nu+\frac{1}{2} \geq 0,
\end{aligned}
$$

and for $n \geq 1$,

$$
\frac{a_{n}}{b_{n}}-c(p)=(n-1)(2 \nu+1-2 p)+\left(\nu+\frac{1}{2}\right) \frac{2 \nu+3}{2 n+2 \nu+1}>0 .
$$

Thus

$$
\lambda_{p, \nu}=\frac{f_{1}\left(x_{0}\right)}{f_{2}\left(x_{0}\right)}=\frac{\sum_{n=0}^{\infty} a_{n}\left(x_{0}^{2} / 4\right)^{n}}{\sum_{n=0}^{\infty} b_{n}\left(x_{0}^{2} / 4\right)^{n}}>\frac{\sum_{n=0}^{\infty} c(p) b_{n}\left(x_{0}^{2} / 4\right)^{n}}{\sum_{n=0}^{\infty} b_{n}\left(x_{0}^{2} / 4\right)^{n}}=c(p),
$$

and

$$
p^{2}+\lambda_{p, \nu}>p^{2}+c(p)=p^{2}+(2 \nu+3)(2 \nu+1-2 p)+\nu+\frac{1}{2}=\xi_{p}^{2}
$$

which proves (4.12) due to Theorem 4.7.

Similarly, we easily check that

$$
\frac{a_{0}}{b_{0}}-\frac{a_{1}}{b_{1}}=2(p-\nu)>0
$$

and for $n \geq 2$,

$$
\begin{aligned}
\frac{a_{n}}{b_{n}}-\frac{a_{1}}{b_{1}} & =(n-1)(2 \nu+1-2 p)-(2 \nu+1) \frac{n-1}{2 n+2 \nu+1} \\
& \geq(n-1)\left(2 \nu+1-2 \frac{(\nu+2)(2 \nu+1)}{(2 \nu+5)}\right)-(2 \nu+1) \frac{n-1}{2 n+2 \nu+1} \\
& =2(2 \nu+1) \frac{(n-1)(n-2)}{(2 \nu+5)(2 n+2 \nu+1)} \geq 0 .
\end{aligned}
$$

Therefore, we have

$$
\lambda_{p, \nu}=\frac{f_{1}\left(x_{0}\right)}{f_{2}\left(x_{0}\right)}=\frac{\sum_{n=0}^{\infty} a_{n}\left(x_{0}^{2} / 4\right)^{n}}{\sum_{n=0}^{\infty} b_{n}\left(x_{0}^{2} / 4\right)^{n}}>\frac{\sum_{n=0}^{\infty}\left(a_{1} / b_{1}\right) b_{n}\left(x_{0}^{2} / 4\right)^{n}}{\sum_{n=0}^{\infty} b_{n}\left(x_{0}^{2} / 4\right)^{n}}=\frac{a_{1}}{b_{1}},
$$

and

$$
p^{2}+\lambda_{p, \nu}>p^{2}+\frac{a_{1}}{b_{1}}=p^{2}+(2 \nu+3)(2 \nu-2 p+1)+2 \nu+1=\theta_{p}^{2},
$$

which proves (4.13). 
Remark 4.12. Since $p+\xi_{p}>0$, Corollary 4.11 implies a new upper bound $G_{p, \xi_{p}}(x)$ for $R_{\nu}(x)$ for $-1 / 2 \leq \nu<p<\nu+1 / 2$. However, the bound $G_{p, \xi_{p}}(x)$ is weaker than $G_{p, q_{\nu}^{*}(p)}(x)$ for $-1 / 2 \leq \nu<p<\nu+1 / 2$ given in [13, Theorem 7]. In fact, we have

$$
\begin{gathered}
q_{\nu}^{*}(p)^{2}-\xi_{p}^{2}=(\sqrt{2(\nu+1 / 2-p)}+\sqrt{(p+1)(2 \nu+1-p)})^{2} \\
-\left[(2 \nu+3-p)^{2}-(3 \nu+11 / 2)\right] \\
=2 \sqrt{2(\nu+1 / 2-p)} \sqrt{(p+1)(2 \nu+1-p)}-\frac{1}{2}(2 p-4 \nu-3)(2 p-2 \nu-1) \\
:=\Phi_{1}(p)-\Phi_{2}(p), \\
\Phi_{1}^{2}(p)-\Phi_{2}^{2}(p)=\frac{1}{2}\left(\nu+\frac{1}{2}-p\right) \Phi_{3}(p),
\end{gathered}
$$

where

$\Phi_{3}(p)=8 p^{3}-4(10 \nu+11) p^{2}+2\left(32 \nu^{2}+60 \nu+15\right) p-(4 \nu+7)(4 \nu-1)(2 \nu+1)$.

Since

$$
\Phi_{3}^{\prime \prime}(p)=8(6 p-10 \nu-11)<8\left(6\left(\nu+\frac{1}{2}\right)-10 \nu-11\right)=-32(\nu+2)<0,
$$

and

$$
\begin{aligned}
\Phi_{3}(\nu) & =(6 \nu+7)(2 \nu+1)>0, \\
\Phi_{3}\left(\nu+\frac{1}{2}\right) & =4(2 \nu+3)(2 \nu+1)>0,
\end{aligned}
$$

by the property of the concave function we have that for $-1 / 2<v<p<$ $v+1 / 2$,

$$
\Phi_{3}(p)>\frac{v+1 / 2-p}{1 / 2} \Phi_{3}(\nu)+\frac{p-\nu}{1 / 2} \Phi_{3}\left(\nu+\frac{1}{2}\right)>0,
$$

which implies that $q_{\nu}^{*}(p)-\xi_{p}>0$, and so $G_{p, q_{\nu}^{*}(p)}(x)<G_{p, \xi_{p}}(x)$ for $x>0$.

Similarly, for $\nu<p<\nu+1 / 2$ there exist some $\nu \in(-3 / 2,-1 / 2)$ such that $p^{2}+\lambda_{p, \nu}$ is positive and explicitly characterized. For example, from Subcase 2.3 we see that for $n \geq 0$,

$$
\frac{a_{n}}{b_{n}}-\frac{a_{1}}{b_{1}}=(n-1)(2 \nu+1-2 p)-(2 \nu+1) \frac{n-1}{2 n+2 \nu+1} \geq 0 .
$$

Then for $\nu \in(-3 / 2,-1 / 2)$ the inequality (4.13) also holds for $x>0$ but the parameter $p$ has to satisfy

$$
\theta_{p}^{2}=(2 \nu+3-p)^{2}-(2 \nu+5) \geq 0,
$$

that is, $v<p \leq 2 \nu+3-\sqrt{2 \nu+5}<\nu+1 / 2$. This can be stated as a corollary. 
Corollary 4.13. Let $-3 / 2<\nu<-1 / 2$ and $\nu_{0}=2 \nu+3-\sqrt{2 \nu+5}$. Then, for $\nu<p \leq \nu_{0}<\nu+1 / 2$ the inequality (4.13) also holds for all $x>0$. In particular, while $-1<\nu<p \leq(\nu+2)(2 \nu+1) /(2 \nu+3)<\nu_{0}$ we have

$$
R_{\nu}(x)<\frac{x}{p+\sqrt{x^{2}+\theta_{p}^{2}}}=G_{p, \theta_{p}}(x), \quad \forall x>0 .
$$

Proof. It remains to prove (4.15). To this end, it suffices to determine the range of $p$ such that $p+\theta_{p} \geq 0$. We easily verify that the function $p \mapsto p+\theta_{p}$ is decreasing on $\left(\nu, \nu_{0}\right]$, and

$$
\left.\left(p+\theta_{p}\right)\right|_{p=\nu}=2(\nu+1)>0, \text { and }\left.\left(p+\theta_{p}\right)\right|_{p=\nu_{0}}=\nu_{0}<0,
$$

which means that there exists a unique $p_{0}=(\nu+2)(2 \nu+1) /(2 \nu+3)$ such that $p+\theta_{p} \geq 0$ for $p \in\left(\nu, p_{0}\right]$, and $p+\theta_{p}<0$ for $p \in\left(p_{0}, \nu_{0}\right]$. Consequently, for $-1<\nu<p \leq p_{0}$ the inequality (4.13) is equivalent to another Amos type one, that is, (4.15) holds for $x>0$. This completes the proof.

Remark 4.14. Corollary 4.13 gives another new upper bound $G_{p, \theta_{p}}(x)$ for $R_{\nu}(x)$ when $\nu<p \leq(\nu+2)(2 \nu+1) /(2 \nu+3)$ and $-1<\nu<-1 / 2$. Clearly, the set of bounds $G_{p, \theta_{p}}(x)$ can be divided into two parts:

$$
\begin{aligned}
& \left\{G_{p, \theta_{p}}(x)\right\} \\
= & \left\{G_{p, \theta_{p}}(x): \nu<p \leq 2 \nu+1\right\} \cup\left\{G_{p, \theta_{p}}(x): 2 \nu+1<p \leq \frac{(\nu+2)(2 \nu+1)}{(2 \nu+3)}\right\} .
\end{aligned}
$$

Comparing $G_{p, \theta_{p}}(x)$ with $G_{p, q_{\nu}^{*}(p)}(x)$ we find that

$$
G_{p, q_{\nu}^{*}(p)}(x)<G_{p, \theta_{p}}(x)
$$

for $\nu<p<2 \nu+1<0$. This shows that the Hornik and Grün's upper bound $G_{p, q_{\nu}^{*}(p)}(x)$ in [13, Theorem 7] is superior to $G_{p, \theta_{p}}(x)$ for $\nu<p \leq 2 \nu+1$. While the upper bound $G_{p, \theta_{p}}(x)$ for $2 \nu+1<p \leq(\nu+2)(2 \nu+1) /(2 \nu+3)$ is a new one.

\section{Acknowledgment}

The two authors would like to express many thanks to the referee for his valuable suggestions and comments. The research of the second author is also supported by ERCEA Advanced Grant 2014 669689-HADE, by the MINECO project MTM2014-53850-P, by Basque Government project IT-641-13 and also by the Basque Government through the BERC 2014-2017 program and by Spanish Ministry of Economy and Competitiveness MINECO: BCAM Severo Ochoa excellence accreditation SEV-2013-0323.

\section{References}

[1] Abramowitz, M., Stegun, I. A.: Handbook of Mathematical Functions with Formulas, Graphs, and Mathematical Tables. National Bureau of Standards, Applied Math. Series 55, Dover Publications, New York and Washington(1972) 
[2] Alenxandrov, M. D., Lacis, A. A.: A new three-parameter cloud/aerosol particle size distribution based on the generalized inverse Gaussian density function. Appl. Math. Comput. 116, 153-165 (2000)

[3] Amos, D. E.: Computation of modified Bessel functions and their ratios. Math. Comp. 28, 239-251 (1974)

[4] Baricz, Á., Neuman, E.: Inequalities involving modified Bessel functions of the first kind II. J. Math. Anal. Appl. 332, 265-271 (2007)

[5] Baricz, Á.: Bounds for modified Bessel functions of the first and second kinds. Proceedings of the Edinburgh Mathematical Society 53, 575-599 (2010)

[6] Baricz, Á.: Turán type inequalities for modified Bessel functions. Bull. Aust. Math. Soc. 82(2), 254-264 (2010)

[7] Baricz, Á., Ponnusamy, S.: On Turán type inequalities for modified Bessel functions. Proc. Amer. Math. Soc. 141, 523-532 (2013)

[8] Baricz, Á.: Bounds for Turánians of modified Bessel functions. Expo. Math. 2015(2), 223-251 (2015)

[9] Bhattacharya, S. K.: Bayesian approach to life testing and reliability estimation. J. Amer. Statist. Assoc. 62, 48-62 (1967)

[10] Bhattacharya, S. K.: Bayesian approach to life testing and reliability estimation. J. Amer. Statist. Assoc. 62, 48-62 (1967)

[11] Biernacki, M., Krzyz, J.: On the monotonicity of certain functionals in the theory of analytic functions. Annales Universitatis Mariae Curie-Sklodowska 9, 135-147 (1955)

[12] Devroye, L.: Simulating Bessel random variables. Statist. Probab. Lett. 57, 249-257 (2002)

[13] Hornik, K., Grün, B.: Amos-type bounds for modified Bessel function ratios. J. Math. Anal. Appl. 408, 91-101(2013)

[14] Joshi, C. M., Bissu, S. K.: Some inequalities of Bessel and modified Bessel functions. J. Aust. Math. Soc. Ser. A 50, 333-342 (1991)

[15] Khazron, P. A., Selesnick, I. W.: Bayesian estimation of Bessel K-form random vectors in AWGN. IEEE Signal Process. Lett. 15, 261-264 (2008)

[16] Kokologiannaki, C. G.: Bounds for functions involving ratios of modified Bessel functions. J. Math. Anal. Appl. 385, 737-742 (2012)

[17] Laforgia, A., Natalini, P.: Some inequalities for modified Bessel functions. J. Inequal. Appl. 2010, Art. ID 253035, 10 pp (2010)

[18] Lushnikov, A. A., Bhatt, J. S., Ford, I. J.: Stochastic approach to chemical kinetics in ultrafine aerosols. J. Aerosol Sci. 34, 1117-1133 (2003)

[19] Nàsell, I.: Schistosomiasis in a community with external infection. Proc. 8th intern. Biometric Conf., Editura Academiei Republicii Socialiste Romania, 123131 (1975).

[20] Nȧsell, I., Hirsch, W. M.: The transmission dynamics of schistosomiasis. Comm. Pure Appl. Math. 26, 395-453 (1973)

[21] Nȧsell, I.: Schistosomiasis in a community with external infection. Proc. 8th intern. Biometric Conf., Editura Academiei Republicii Socialiste Romania, 123131 (1975).

[22] Nàsell, I., Hirsch, W. M.: The transmission dynamics of schistosomiasis. Comm. Pure Appl. Math. 26, 395-453 (1973) 
[23] Nȧsell, I.: Rational bounds for ratios of modified Bessel functions. SIAM J. Math. Anal. 9, 1-11(1978)

[24] Neuman, E.: Inequalities involving modified Bessel functions of the first kind. J. Math. Anal. Appl. 171, 532-536(1992)

[25] Petropoulou, E.: Bounds for ratios of modified Bessel functions. Integral Transforms and Special Functions 9(4), 293-298 (2000)

[26] Pólya, G., Szegö, G.: Problems and Theorems in Analysis I: Series, Integral Calculus, Theory of Functions. Classics in Mathematics, Springer-Verlag, BerlinHeidelberg-New York(1998)

[27] Robert, C.: Modified Bessel functions and their applications in probability and statistics. Statist. Probab. Lett. 9(2), 155-161 (1990)

[28] Segura, J.: Bounds for ratios of modified Bessel functions and associated Turántype inequalities. J. Math. Anal. Appl. 374, 516-528 (2011)

[29] Simpson,H. C., Spector, S. J.: Some monotonicity results for ratios of modified Bessel functions. Quart. Appl. Math. 42, 95-98 (1984)

[30] Simpson, H. C., Spector, S. J.: On barelling for a special material in finite elasticity. Quart. Appl. Math. 42, 99-105 (1984)

[31] Tan, S., Jiao, L.: Multishrinkage: Analytical form for a Bayesian wavelet estimator based on the multivariate Laplacian model. Optim. Lett. 32, 2583-2585 (2007)

[32] Thiruvenkatachar, V. R., Nanjundiah, T. S.: Inequalities concerning Bessel functions and orthogonal polynomials. Proc. Ind. Acad. Sci. Sect. A 33, 373384 (1951)

[33] Watson, G. N. : A Treatise on the Theory of Bessel Functions. Cambridge University Press, Cambridge (1922)

[34] Xia, F. L., Yang, Z. H., Chu, Y. M.: A new proof for the monotonicity criterion of the quotient of two power series on the infinite interval. Pacific Journal of Applied Mathematics 7(2), 97-101(2016)

[35] Yang,Z. H., Chu, Y. M., Wang,M. K.: Monotonicity criterion for the quotient of power series with applications. J. Math. Anal. Appl. 428, 587-604 (2015)

[36] Yuan, L., Kalbfleisch, J. D.: On the Bessel distribution and related problems. Ann. Inst. Statist. Math. 52(3), 438-447 (2000)

\author{
Zhen-Hang Yang \\ ${ }^{1}$ Department of Mathematics \\ Beijing Jiaotong University \\ Beijing 100044 \\ China \\ e-mail: yzhkm@163.com \\ ${ }^{2}$ Customer Service Service Center \\ State Grid Zhejiang Electric Power Research Institute \\ Hangzhou 310009, Zhejiang \\ China
}




\author{
Shen-Zhou Zheng \\ ${ }^{1}$ Department of Mathematics \\ Beijing Jiaotong University \\ Beijing 100044 \\ China \\ e-mail: shzhzheng@bjtu.edu.cn \\ ${ }^{2}$ BCAM-Basque Center for Applied Mathematics \\ Alameda de Mazarredo 14 \\ 48009 Bilbao \\ Spain
}

